\title{
III. THE SPECTRAL SEQUENCE AND ITS ANOMALIES
}

\author{
August I7, I948. I0.30 a.m. \\ Chairman, Prof. P. Swings \\ r. OBJECTIVE PRISM SPECTRA
}

\author{
By J. J. NASSAU \\ Warner and Swasey Observatory, Case Institute of Technology, Cleveland, Ohio
}

This report deals mainly with the study of spectra secured with a $4^{\circ}$ objective prism attached to the 24-36-inch Schmidt-type telescope of the Warner and Swasey Observatory of the Case Institute of Technology. The dispersion at $\mathrm{H}_{\gamma}$ is $283 \mathrm{~A} . / \mathrm{mm}$. Spectra obtained with a $2^{\circ}$ objective prism will be dealt with briefly. We will consider first spectra secured by means of long exposures intended to be used for statistical studies; second, spectra of relatively short exposures for detailed study of individual stars and, third, infra-red spectra for the classification of low-temperature stars.

Part $I$. Here we are concerned with spectra of stars of limiting photographic magnitude of 12.5 secured with an exposure of 30 minutes on plates of intermediate speeds. From such spectra stars are classified in four sub-classes in each spectral class. In the case of the $\mathrm{B}$ stars five classes are assigned. Between $\mathrm{Fo}$ to $\mathrm{K}_{5}$ we are able to distinguish four luminosity classes for stars one magnitude brighter than the limiting magnitude of the plate; for the other spectral classes three. The probable error of one estimate of spectral type is \pm 0.12 and for one estimate of luminosity class \pm 0.30 . The interval between I, III, IV and V (Morgan, Keenan and Kellman notation) is taken as one unit with the exception of the interval between III and IV, which is taken as one half unit. Although it is possible to classify I $a$ stars, they are not included in the calculation of the probable errors.

At present it appears that it is possible to increase the accuracy in the classification of the $\mathrm{B}$ stars by adding a step between $\mathrm{B}_{2}$ and $\mathrm{B} 5$.

Spectra secured with the $2^{\circ}$ prism are employed primarily in order to avoid overlaps in crowded regions. They reach stars about $0 \cdot 4$ of a magnitude fainter than with the $4^{\circ}$ prism and compare in accuracy with the faint stars of this prism.

Part II. Spectra from which detailed study of individual stars is possible are made with short exposures on intermediate or slow emulsion plates and are from 0.3 to $0.4 \mathrm{~mm}$. wide. From such plates the sky fog is materially decreased, and seeing and differential refraction effects are diminished. The resulting spectra show clearly all the features upon which the criteria of temperature and luminosity classifications are based. As, for example, in the case of the F-type stars between the spectral region of $\lambda 3706$ and $\mathrm{H}_{\gamma}$ 30 lines and line-blends are recognized and used in the classification. Comparison with standards are made by means of visually drawn profiles which show the depth and width of such lines for all stars between $F_{0}$ to $\mathrm{K}_{5}$ and for all four or five luminosity classes. Assignment to a class becomes certain provided conflicting criteria do not exist and other peculiarities are not present.

The classification of the normal main sequence $A$ stars is relatively simple as the growth of the $\mathrm{K}$ line with respect to hydrogen is a safe criterion, provided the G-band and other metallic lines are not present. Our practice has been to give two classes to such stars, although according to Greenstein the $\mathrm{F}$ class seems more appropriate. The recognition of peculiar A stars such as silicon and strontium stars is also possible. For luminosity classification, the narrowing of the $\mathrm{H}$ lines and the strength of certain lines and blends are employed.

The accurate classification of B stars both in the temperature and luminosity classes seems probable. 
A survey with high-quality spectra of a $12^{\circ}$-belt along the galactic equator from longitude $333^{\circ}$ to $197^{\circ}$ is now in progress as a co-operative project between the Yerkes and Warner and Swasey Observatories. It aims to reach stars to the roth photographic magnitude with high-quality spectra for the discovery of B stars and other stars of high luminosity.

Part III. The spectral region between $\lambda 6800$ and $\lambda 8800$ with dispersion at the $\mathrm{A}$ band of about $\mathrm{r} 700 \mathrm{~A} . / \mathrm{mm}$. with the four-degree prism and $3400 \mathrm{~A} . / \mathrm{mm}$. with the two-degree prism proved successful in classifying M-type, carbon, and S-type stars. The classification of the $\mathrm{M}$ stars is based solely on the TiO bands. The probable error of a single estimate is equal to \pm 0.3 decimal division. $M$ stars as faint as 14.4 infra-red magnitude are classified. No means for establishing luminosity criteria have been found. Carbon stars are identified from the band of $\mathrm{CN}$, while a new band, $\lambda 795^{\circ}$, which according to Keenan is due to $\mathrm{LaO}$, is utilized in the identification of $\mathrm{S}$ stars.

\section{CLASSIFICATION SPECTRALE DES ETOILES DES PREMIERS TYPES SPECTRAUX D'APRES LA GRANDEUR ET LA POSITION DE LA DISCONTINUITE DE BALMER}

Par Daniel Barbier et Daniel Chalonge

Institut d'Astrophysique et Observatoire de Paris

\section{RÉSUMÉ}

I. Rappel de la définition des grandeurs utilisées $D$ et $\lambda_{1}$.

II. La classification spectrale (type spectral et magnitude absolue) des étoiles normales par leur structure et leur composition chimique dépend de deux paramètres $T_{e}$ et $g$. Il en est de même de $D$ et $\lambda_{1}$. Il est donc possible de faire une classification spectrale à partir de ces deux derniers paramètres. L'intérêt d'une telle classification provient de ce qu'elle ne fait intervenir qu'un seul corps, l'hydrogène, constituant principal des atmosphères stellaires.

III. Dans l'état actuel de la théorie on doit rechercher par une méthode empirique les relations existant entre le type spectral, la magnitude absolue et $D$ et $\lambda_{1}$. Le nombre des étoiles observées est encore petit et les résultats obtenus doivent être considérés comme préliminaires.

IV. La corrélation entre le type spectral ainsi déterminé et le type spectral de Harvard est bonne. Les désaccords ne sont pas plus grands qu'entre deux classifications spectrales classiques.

V. La corrélation entre les magnitudes absolues ainsi déterminées et les magnitudes absolues provenant des parallaxes trigonométriques, des parallaxes de groupe etc. est satisfaisante. On peut même constater qu'à l'intérieur d'un type spectral déterminé $\left(A_{0}\right)$ les nouvelles parallaxes absolues sont plus précises que les parallaxes spectroscopiques classiques.

\section{BIBLIOGRAPHIE}

Daniel Barbier et Daniel Chalonge. Annales d'Astrophysique, 2 (1939), p. 254.

Daniel Barbier et Daniel Chalonge. Annales d'Astrophysique, ro (I947), p. 195.

Discussion. Dr B. Lindblad pointed out the Stockholm observations of a strong Balmer discontinuity in super-giants. 


\title{
3. ANOMALIES IN THE SPECTROPHOTOMETRIC CRITERIA OF STELLAR LUMINOSITY IN THE SPECTRAL SEQUENCE OF THE HYADES
}

\author{
By Jöran M. RAMberg \\ Stockholm's Observatory, Saltsjöbaden, Sweden
}

At the Stockholm Observatory at Saltsjöbaden we have long been occupied with applications of the spectrophotometric parallax method originally introduced by Prof. B. Lindblad and developed by him and his collaborators at the Uppsala and Stockholm Observatories. At the Stockholm Observatory the work is mainly based on the $40 \mathrm{~cm}$. Zeiss astrograph combined with an objective prism of the same aperture giving a dispersion on the plate of $\mathrm{r} \cdot 8 \mathrm{~mm}$. between $\mathrm{H} \gamma$ and $\mathrm{H} \epsilon$. The plates are taken without lateral widening of the spectral images and are registered with a broad diaphragm by means of a Koch-Goos self-recording microphotometer.

The spectrophotometric parallax method consists essentially in the determination of the monochromatic intensities of the stars for a number of selected wave-lengths. From the reduced measurements of the tracings of the objective prism spectra a number of spectral quantities are obtained, defined as follows:

For the early-type stars: the intensities of $\mathrm{H} \gamma$ and $\mathrm{H} \delta$,

$$
\begin{aligned}
& \mathrm{H} \gamma=m \mathrm{H}_{\gamma}-\frac{1}{2}\left(m_{4400}+m_{4260}\right), \\
& \mathrm{H} \delta=m \mathrm{H} \delta-\frac{1}{2}\left(m_{4140}+m_{4050}\right) .
\end{aligned}
$$

For the late-type stars: the break of intensity at the $G$-band, $g$, the cyanogen equivalent, $c$, and the intensity of the calcium line $\lambda 4227$,

$$
\begin{aligned}
g & =m_{4260}-m_{4360}, \\
c & =m_{4180}-m_{4260}, \\
\lambda_{4227} & =m_{4227}-\frac{1}{2}\left(m_{4260}+m_{4200}\right) .
\end{aligned}
$$

In addition to the spectrophotometric measurements, the spectra are classified on the basis of those characteristics which are most readily identified in our spectra.

For the present I am occupied in a spectrophotometric survey of selected regions in and around the central band of the Milky Way in Lacerta, Cepheus, and Auriga. Some time ago I made a survey of the same kind of the central part of the region of the Hyades.* The test of the spectrophotometric criteria of stellar luminosity on the physical members of the Hyades may be of interest here.

The investigation comprised in all forty-eight physical members of the cluster. The Hertzsprung-Russell diagram of the investigated cluster members is shown in Fig. I. The absolute magnitudes of the cluster members were computed, adopting Smart's $\dagger$ value of the parallax of the cluster

$$
\pi=0 " 028 \text {. }
$$

The dotted line in the figure defines the ridge-line of the normal main series as determined from the Mount Wilson catalogue of spectroscopic parallaxes. The line is drawn with attention paid to the differences in the Stockholm system of spectral classification from that of the Mount Wilson Observatory. It is seen from the figure that, apart from the latest type dwarfs, the members of the Hyades appear to be systematically fainter than the random stars of the main series. The systematic departure of the cluster members from random stars may of course partly be explained by the fact that the average absolute magnitudes obtained from the Mount Wilson catalogue refer to scattered stars brighter than a certain magnitude, but the corrections to the element of volume for the Mount Wilson system of absolute magnitudes are likely to be small. The departure considered may also be partly ascribed to uncertainty in the parallax of the Hyades cluster, but

* Ramberg, J. M., 'A Spectrophotometric Study of the Regions of the Hyades and Praesepe', Stockholm Annaler, Band 13, no. 9, I94I.

† Smart, W. M., 'The Moving Cluster in Taurus', M.N. 99, I68, 1939. 


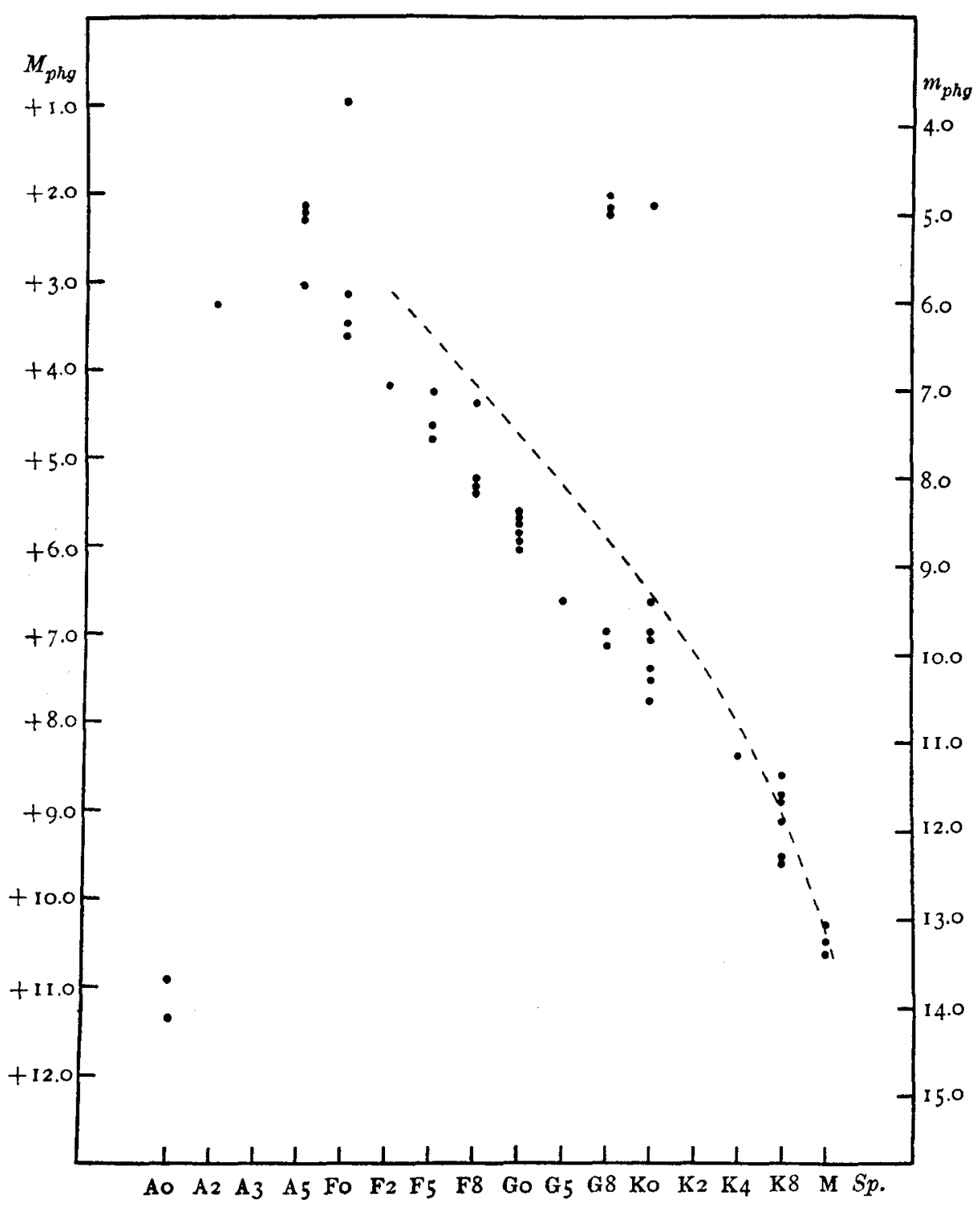

Fig. 1. Hertzsprung-Russell diagram of the members of the Hyades included in the present investigation. The dotted line defines the ridge-line of the normal main series.

even this seems able to explain only a small amount of the systematic difference observed. Furthermore, the fact that the latest type dwarfs among the cluster members appear to be quite normal argues against the assumption that Smart's value of the parallax is too great.

In Fig. 2 the hydrogen intensities of the A-F-type Hyades stars are drawn against the photographic absolute magnitudes. In the same figure a luminosity diagram for the spectral classes $\mathrm{B}-\mathrm{A}_{3}$ and $\mathrm{F}_{2}-\mathrm{F} 8$ and valid for scattered stars is copied. The diagram was deduced by the author on the basis of measurements published by Lindblad, Schalén, and Öhman. 
In Fig. 3 the measurements of the cyanogen absorption in the dGo-dM stars belonging to the cluster have been plotted. In the same figure the curve showing the connection between the cyanogen absorption and the absolute photographic magnitude in scattered stars is copied. In Fig. 2 as well as in Fig. 3 the deviation of the cluster members from the scattered stars is striking. The break of intensity at the $G$ band and the intensity of the calcium line $\lambda 4227$ show the same deviations. Accordingly, all spectrophotometric results

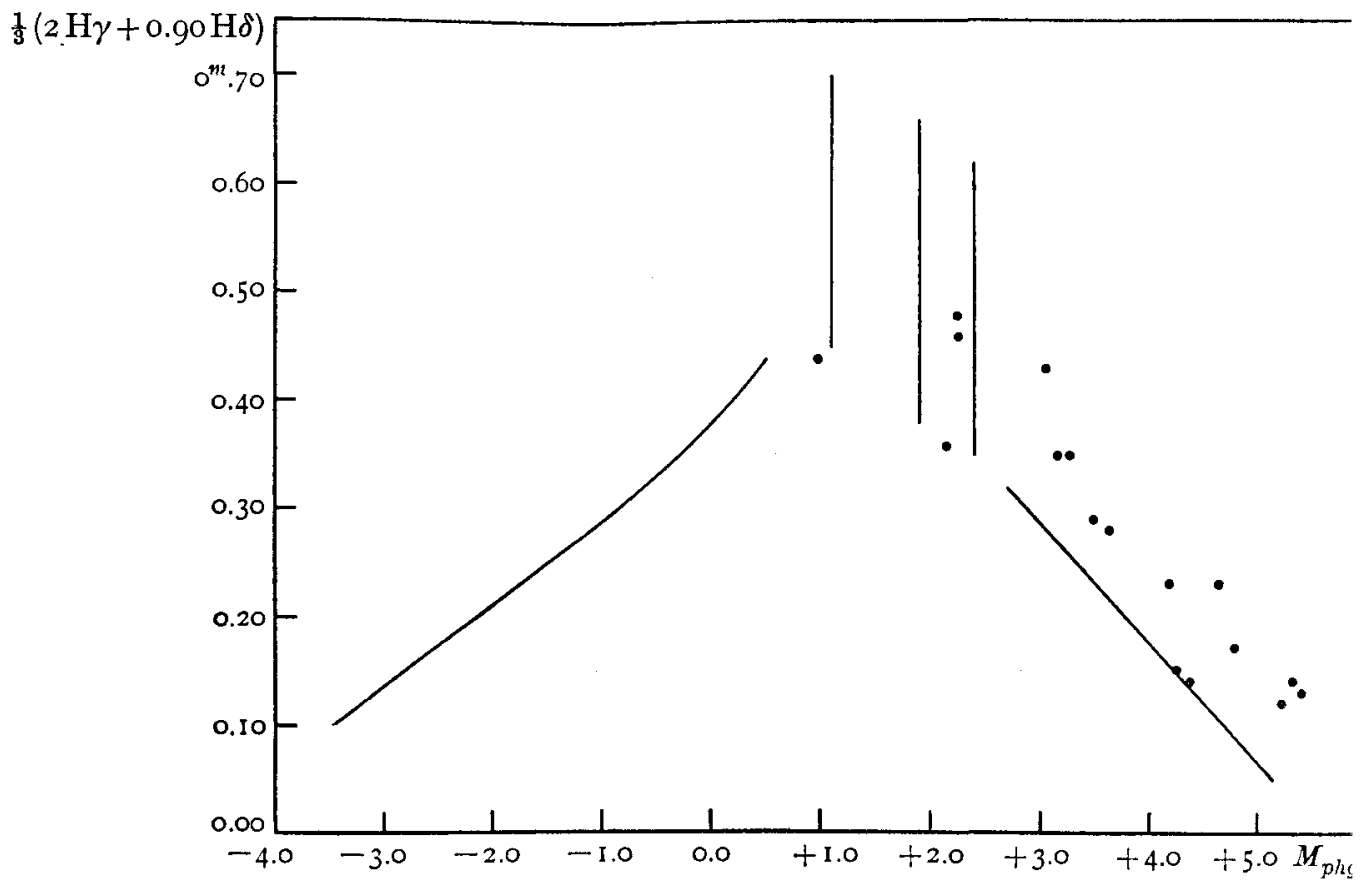

Fig. 2. The relation between the hydrogen line intensity $\mathrm{I} / 3(2 \mathrm{H} \gamma+0.90 \mathrm{H} \delta)$ and the photographic absolute magnitude for the A-F-type members of the Hyades. The diagram valid for random stars is copied in the figure.

argue in favour of the reality of the phenomenon appearing in Fig. I. The members of the Hyades belonging to the main series are systematically fainter than random stars of corresponding spectral types. The departure in magnitude is maximal early in the spectral sequence and seems to decrease with advancing spectral type and to disappear in late $\mathrm{K}$ and $\mathrm{M}$ dwarf stars.

It is possible that the shift observed in the absolute magnitudes of the Hyades in relation to random stars corresponds to some spectral peculiarities in one way or another. No such peculiarities could, however, be traced in the astrographic spectra considered. Judging from the astrographic spectra the members of the Hyades must be characterized as typical dwarf stars without any peculiarities with regard to the distribution of intensity in the spectrum. This fact seems to force upon us the opinion that within each spectral class of the main series we have a rather considerable cosmic dispersion.

The fact that the very late-type dwarfs among the Hyades members appear to be 'normal' in respect of absolute magnitude may be explained by assuming that the cosmic dispersion in luminosity within each spectral class of late $K$ and $M$ dwarfs is on the whole small. The view is supported also by the Hertzsprung-Russell diagram of the nearest stars. Furthermore, there is to be noted in this connection the result found by Kuiper*

* Kuiper, G. P., 'On the Hydrogen Content of Clusters', Ap. J. 86, I76, I937.

$$
446
$$


that the main sequences of different clusters are crowded closely together in the lower part of the main series.

The results obtained argue in favour of the opinion that the main series is built up of different star families among which the members of the Hyades cluster form one. Early in the main series the families deviate considerably in absolute magnitude but converge with advancing spectral type.

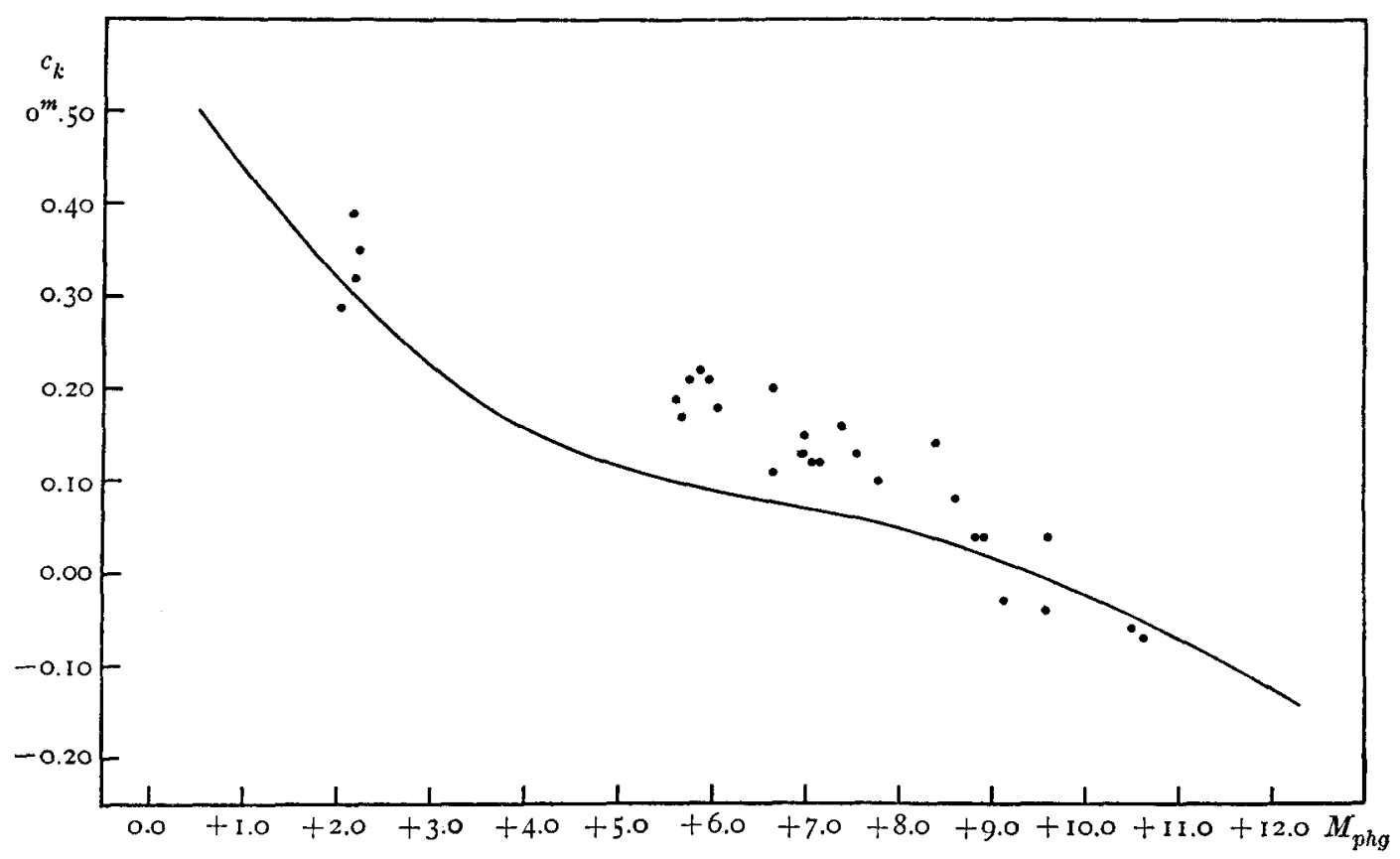

Fig. 3. The relation between the reduced cyanogen equivalent $c_{k}$ and the photographic absolute magnitude for the G-M-type members of the Hyades. The curve valid for random stars is copied in the figure.

It is obvious that the spectrophotometric criteria of stellar luminosity in their present state do not enable us to distinguish between the different sub-classes of dwarfs in a direction perpendicular to the main series. It seems highly desirable that wide dispersion spectra of bright cluster members and of stars with well-determined trigonometric parallaxes are photometrically investigated with respect to luminosity criteria. Work along this line is going on at the Stockholm Observatory.

\section{Discussion}

R. E. WILson. Recent studies of the motions of the Hyades at Mt Wilson show that Smart's parallaxes of these stars are definitely too large. It is also known that the Mt Wilson absolute magnitudes for the $\mathrm{A}$ and $\mathrm{F}$ stars are too bright by at least 0.5 magnitude. Comparisons between these two sets of data necessarily lead to erroneous conclusions regarding the luminosities of the Hyades stars. If the absolute magnitudes derived recently from new cluster parallaxes in the Hyades are compared with those derived by Hopmann for the stars with best-determined trigonometric parallaxes the maximum mean separation of the two Hertzsprung-Russell main sequences is of the order of 0.15 magnitude.

The difference in the dispersion in $M$ between the early-and late-type stars has recently been explained through the work of Dr O. Eggen at the Washburn Observatory. His 
observations of photoelectric magnitudes of the brighter stars in the Hyades and several other open clusters (observations which show remarkably small dispersion about a mean $\mathrm{H}-\mathrm{R}$ sequence) establish a split in the sequence for the early-type stars. The brighter branch contains the metallic line stars and others which show in their spectra differences from the characteristics of the main sequence stars. When this split is taken into consideration, the dispersion in $\mathrm{M}$ of the early-type stars on the main sequence is essentially the same as that of the late types.

\section{PECULIARITIES FREQUENTLY ENCOUNTERED IN SPECTRA OF SHORT DISPERSION}

By A. N. Vyssotsky

\section{Leander McCormick Observatory, Charlottesville, Virginia}

The McCormick objective prism plates, which cover the sky from the north pole to declination $-15^{\circ}$, show classifiable spectra down to twelfth photographic magnitude. The dispersion at $\mathrm{H} \gamma$ is about $300 \mathrm{~A} . / \mathrm{mm}$. Because of the steep colour curve of the instrument the portion of the spectrum between $\lambda_{4100}$ and $\lambda_{4000}$ remains unobserved.

While relatively few features are available for temperature classification with our instrumental arrangement, additional details are frequently visible. The significance of only a few of these is understood. The following list contains the peculiarities most frequently encountered.

(I) Simultaneous strengthening of the $\mathrm{G}$ band and $\mathrm{H} \gamma$ in certain $\mathrm{G}$ stars. We have correlated this feature statistically with larger than average proper motions.

(2) A line or group of lines near $\lambda 4300$ in A stars. So far we do not know the significance of this 'pseudo-G band'.

(3) Very narrow $\mathrm{H}$ and $\mathrm{Ca}$ II lines in certain F stars, which thus resemble RR Lyrae stars but are not known to be variable.

(4) A strong narrow line near $\lambda_{4} \mathrm{I} 75$ in certain $A$ and F stars. If this is the Ti II-Y II line which appears in the super-giants of these classes, it is difficult to explain why these spectra are occasionally found in high galactic latitude.

(5) A depression in the continuous spectrum of $\mathrm{K}$ stars between $\lambda 4400$ and $\lambda 4500$ which is sometimes very pronounced and gives the impression of a band.

(6) Strong CN absorption at $\lambda_{42} I_{5}$ is conspicuous in $\mathrm{K}$ super-giants and in certain $\mathrm{K}$ giants on a given plate. What differentiates these $\mathrm{K}$ giants from the others in which cyanogen is not conspicuous is not known. In this connection sharp $\mathrm{CN}$ absorption at $\lambda_{42} \mathrm{r}$ distinguishes very early-type $\mathrm{R}$ stars which otherwise resemble $\mathrm{G} 5$ stars.

(7) Finally, we do not know the physical cause of the 'Lindblad depression' between $\lambda 4227$ and $\lambda 43$ Io in all $\mathrm{M}$ dwarfs.

\section{Discussion}

Dr B. LindBlaD. When the absorption due to the CN band is measured with a microphotometer, it is found to be a good criterion for an absolute magnitude classification of $G$ and $K$ stars, with the exception of the stars of high velocity.

Dr L. Gratton. On high-dispersion spectra of $\mathrm{K}$ giants the close doublets which form the rotational components of the $\mathrm{CN}$ band have a very different aspect in high-velocity stars as compared with ordinary stars. For instance, in $\alpha$ Bootis the two components are almost blended, whereas in ordinary giants they are very well separated; the same is true also for $\gamma$ Leonis and $\eta$ Cephei. On the other hand, there are also some ordinary stars, like $\beta$ Aquilae, which show the same characteristics.

Dr P. Swings. Is the strengthening of the $\mathrm{G}$ band and $\mathrm{H} \gamma$ in certain $\mathrm{G}$ stars related to the high-velocity $\mathrm{CH}$ stars? Of course, if hydrogen is enhanced, we may expect $\mathrm{CH}$ to have a chance also to be enhanced.

Is the 'pseudo-G band' near $\lambda_{4300}$ present in early or late A's? If there are stars with abnormal Eu II, Dy II or such things, we may possibly expect others with abnormal Eu III, or Dy III. Observations of these spectra with higher resolution are much needed. 
Is the depression near $\lambda 445^{\circ}$ in $\mathrm{K}$ stars symmetrical? How is the $\mathrm{G}$ band in these $\mathrm{K}$ super-giants?

In the absence of any other interpretation of the 'Lindblad depression' in M dwarfs, I think that Lindblad's own original interpretation is still worth considering (quasimolecule of $\left(\mathrm{Ca}_{2}\right)$.

Dr Vyssotsky. The depression near $\lambda 4450$ in $\mathrm{K}$ stars is practically symmetrical. In $\mathrm{K}$ super-giants the $\mathrm{G}$ band is faint but quite normal. The 'pseudo- $\mathrm{G}$ band' is present in the early A stars.

\section{THE PLACE OF THE WOLF-RAYET STARS IN THE STELLAR SEQUENCE}

By C. S. Beals

\section{Dominion Astrophysical Observatory, Victoria, B.C., Canada}

The spectrum of a typical Wolf-Rayet star consists of a continuous spectrum on which are superimposed numerous broad emission bands due to atoms of high ionization potential. The breadth of the bands may be anywhere from I5 to Ioo A. and their combined width and intensity is often such as to obscure the underlying continuous spectrum.

An analysis of the atoms present in Wolf-Rayet spectra indicates that the stars are divided into two parallel sequences which are designated as the Carbon Sequence and the Nitrogen Sequence respectively. The Carbon Sequence exhibits bands due to $\mathrm{He} \mathrm{I}$, He II, C II, C III, C IV, O II, O III, O IV, O V and O VI with nitrogen weak or absent. The Nitrogen Sequence has bands due to He I, He II, N III, N IV and N V with carbon and oxygen weak or absent.

A good criterion of excitation is provided by the $\mathrm{He}$ I/He II ratio making use of the lines $\lambda_{5875}$ of $\mathrm{He} \mathrm{I}$ and $\lambda_{54 \mathrm{II}}$ of $\mathrm{He}$ II. Making use of this, as well as a number of subsidiary ratios, the stars were divided into a number of classes as outlined in the report of Commission 29 of the I.A.U. in I938. The distribution of the classes is as follows, where WC refers to the Carbon Sequence and WN to the Nitrogen Sequence.

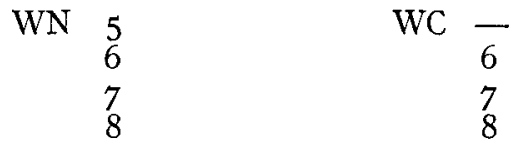

The two sequences are roughly parallel as regards excitation.

The two sequences, as might be expected, are also parallel in temperature. Values of photoelectric temperatures derived by Zanstra's method indicate values ranging from 60,000 to $110,000^{\circ}$. These values refer, of course, to the atoms of highest ionization potential and there is undoubtedly some stratification in the atmospheres.

Diameters computed on the basis of the above temperatures and the observed apparent magnitudes are of the order of $I \cdot 0$ to $4 \cdot 0 \odot$. Since these computations did not take into account the effect of general absorption by interstellar material, they are probably too low and may require to be increased by a factor of two or more.

An astrophysical problem of great interest is whether the Wolf-Rayet stars represent an extension to earlier spectral types of the absorption O's or whether they constitute a separate sequence.

It is doubtful whether the observations at present available are sufficient to decide this question. It may be noted, however, that in a number of $\mathrm{O}$-type stars of earliest spectral type, lines due to He II, N III and C III appear in emission and it is lines of C III and N III which appear strongly in the Wolf-Rayet stars of lowest excitation. It may further be remarked that there is a complete lack of absorption line stars of type earlier than $\mathrm{O}_{5}$. It is perhaps possible that the nuclei of certain planetary nebulae which do not show emission bands may correspond in excitation to this part of the stellar sequence, but unfortunately, with the exception of certain objects of relatively low excitation, the presence or absence of an absorption spectrum associated with these stars has not been determined. 
It is possible that a clear understanding of the physical relations between the WolfRayet stars and the rest of the sequence will only come about as a result of theoretical studies of the origin of Wolf-Rayet emission.

The theory now most commonly accepted is that the emission is a consequence of the continuous ejection of atoms from the stellar surface, although some astronomers are not convinced that this is a satisfactory explanation.

Even if this general mechanism be accepted, the cause of the ejection of atoms is still unknown. Presumably it arises as a consequence of some combination of mass, diameter, surface temperature and rate of energy generation in the star. Little, if any, theoretical work has been done on this problem and our knowledge of the fundamental physical processes responsible for the unique characteristics of these stars is very inadequate.

For the more numerous stars of the main sequence, models of inner structure and atmosphere have been proposed which account satisfactorily for the principal observed spectral features. This is not the case for the Wolf-Rayet stars, and until it is possible by theoretical and observational studies to determine the particular physical characteristics of a stellar model which is responsible for the unique features of Wolf-Rayet spectra, the relationship of these objects to the rest of the stellar sequence is likely to remain obscure.

In the same connection it is also highly desirable to examine in greater detail the nuclei of planetary nebulae of high excitation. Such observations are rendered difficult by the faintness of the stars and by the superimposed nebular line spectrum. It is to be hoped that observations of sufficient dispersion may eventually be possible with the aid of powerful telescopes, and when such observations are available they should help to clarify the general problem of the classification of the hotter stars.

\section{ANOMALIES IN THE EARLIEST SPECTRAL TYPES}

\section{By P. Swings \\ Institut d'Astrophysique, Liége (Belgique), and Yerkes Observatory, Williams Bay, Wis. (U.S.A.)}

The spectral classification in types and luminosities is based on the absolute or relative intensities of the absorption or emission lines of one or several atoms or molecules. The absorption or emission intensities, or more generally the profiles, result from the combined effects of absorption and emission in the stellar atmosphere. Certain geometrical, physical and dynamical factors may affect the intensities of emission or absorption of certain lines, rendering them anomalous in the sense that they differ from those of most stars having otherwise the same general characteristics. Since these factors may thus upset the spectral classification or even render it precarious or erroneous, it is important to gain a general understanding of them. Such a study will help in realizing the full meaning of normal intensities, hence of the normal classification. Actually one may wonder whether any star may be regarded as entirely free of peculiarities, or at least of a predisposition to develop them from time to time.

The factors introducing spectral anomalies are best studied in the case of conspicuously peculiar stars. In such cases, the mechanisms producing ionization and excitation must be examined individually. My purpose is to mention some of the factors affecting the spectra of early-type stars. All the objects considered in this report possess extended shells. There is a wide variety of such stellar shells, corresponding to great ranges in conditions of temperature and density.

The Wolf-Rayet stars. Beals's outstanding contributions to these stars gave rise to the classification in WC and WN stars, officially adopted by the I.A.U. at its I938 meeting. Recent investigations make it desirable to complete this classification. It has been shown recently that C IV is present in early WN stars, reaching its maximum in WN 6 , but being still present in WN 7. On the other hand, no nitrogen line has been found so far 
in typical WC stars which are not nuclei of planetary nebulae. Special difficulties arise in the classification of these nuclei of WR type for the following reasons:

(a) many reveal lines of $\mathrm{C}, \mathrm{N}$ and $\mathrm{O}$ simultaneously, with similar intensities (Ex.: nuclei of NGC 6543, IC 4997, IC 4I8);

(b) the lines are often relatively sharp;

(c) it is often difficult to separate the nuclear and nebular contributions in the $\mathrm{H}, \mathrm{He} \mathrm{I}$, He II and other lines.

For these and other reasons, the classification of the WR stars should profitably be re-examined. Suggestions on this matter are made in the complete paper.

Great care has to be exercised in the interpretation of the Zanstra temperatures obtained for the WR stars, because of the stratification effects and of the photo-ionization by emission lines.

The Of stars. The spectra of these stars are superpositions of typical spectra of absorption $\mathrm{O}$ stars and of shell lines of high excitation, principally $\mathrm{N}$ III and He II; sometimes also of $\mathrm{H}, \mathrm{He} \mathrm{I}, \mathrm{N}$ IV, N V and Si IV. All varieties are observed between pure absorption $\mathrm{O}$ stars and pure WR stars. There is also a wide variety in the relative intensities of the N III and C III lines, going from Of shells with strong N III and no C III, to Of shells with strong C III and weak N III.

A very striking selection is found among the emission and absorption shell lines observed in Of stars. The observed selection has no connection either with the metastability of certain spectral terms or with the excitation potentials. This selection decreases when the emission and absorption in the shell increase. The selection has actually disappeared in WR atmospheres.

Anomalous intensities. Anomalies are found for practically every atom. Their interpretation reveals a variety of physical processes which are able to affect considerably the relative intensities or, more generally, the profiles of certain lines within a specific atom. It is useful to examine each atom individually. A few general results are:

(a) Monochromatic fluorescence due to wave-length coincidences enhances certain lines in O III, N III, He II and O I (Bowen's fluorescence mechanism).

(b) Fluorescence excited by the underlying continuous radiation (sometimes with absorption or emission lines) modifies the intensities in He I, N III, N IV, C III, Si III and other atoms. (Example: the singlet/triplet intensity ratio of He I.)

(c) The mechanisms $(a)$ and $(b)$ depend greatly on dynamical factors, such as ejection, rotation and turbulence, since the wave-length coincidences are affected by the radial velocity displacements within the atmosphere. For example, axial rotation should be considered not only as a dynamical agent in the formation of a shell, but also as a mechanism influencing the fluorescence excitation and the expansion which might possibly result from the corresponding radiation pressure. The absence of Bowen's fluorescence mechanism on O III in WR stars is probably a result of velocity effects.

(d) Elements such as Ca II and Si II reveal the abnormal populations of certain levels due to absorption of radiations (such as $\mathrm{L}_{\alpha}$ ) beyond the ionization limits;

(e) Elements such as $\mathrm{He}$ I, Fe II, Fe III, Ni II, Mg II, etc., reveal striking effects due to the geometrical dilution of the exciting radiation. It may happen that the only strong sharp shell line in the spectrum is $\lambda 3889 \mathrm{He} I$.

The presence of absorption or emission lines (or of continua at the series limits) in the underlying radiation affects considerably the excitation and ionization (and dissociation in late-type stars). It gives rise to anomalous distributions among the various stages of ionization. Coupled with stratification effects (somewhat along the lines of Strömgren's zones), these mechanisms explain extremely abnormal relative intensities.

Since the fluorescence excitation in the shell depends on the velocities of the atoms, different shell lines of a specified atom (also lines belonging to different atoms) may give different profiles and radial velocities, both of the absorption and of the emission components. Actually the physical interpretation of the profiles and of the measured line displacements in shell stars is a matter of extreme complexity. For example, the radial velocities of the He I triplets may differ from those of the singlets. 
Differences in relative abundances of the elements should be considered only as a last resort. In fact, great caution is required when trying to estimate the abundances of elements in peculiar stars, including novae.

\section{Discussion}

Dr ZANSTRA. The temperature of Wolf-Rayet stars has no clear meaning when the optical depth in the ultra-violet is too small or too large. In both cases the theory of recombination will predict wrong values of the temperature.

Dr Ambartsumian. The theory of extended atmospheres leads to large departures from a black-body energy curve. On this account, too, the temperature loses its usual significance.

\section{ON THE PATCHY STRUCTURE OF THE INTERSTELLAR ABSORBING LAYER}

\section{By V. Ambartsumian}

Burakan Observatory, near Erevan, Armenia, U.S.S.R.

More than ten years ago it was clearly brought out that the interstellar absorbing medium has a very irregular, patchy structure. During the last years we in the Soviet Union have done some work with the purpose of studying more in detail the structure of the system of absorbing clouds and of obtaining some numerical parameters describing this variety of clouds.

The first question to be answered is: It is known that each of the luminous diffuse nebulae is as a rule illuminated by some high-luminosity star. Are the stars and the corresponding nebulae connected genetically and dynamically or is this connection occasional, due to the fact that the star and nebula meet together by chance during their galactic motion and then separate again?

In order to solve this question it was decided to compare with observations a very important conclusion that can be reached from the hypothesis of the chance meeting.

It is clear that each star can illuminate around itself only a sphere of definite radius in the sense that the cloud situated within this sphere will attain sufficient illumination to be observed as a bright diffuse nebula. Evidently, the radius of such a sphere will be proportional to the square-root of the luminosity of the star.

Let us take a certain volume $V$ of the galactic space. Stars of different spectral types and luminosities will appear in this volume. Let us imagine the spheres illuminated by these stars. Knowing the luminosity function and the star density for each spectral class we can immediately obtain the total volume illuminated by stars of each spectral type

$$
\text { O, Bo, BI, B2-9, A, F, G, K, M. }
$$

In the case of the chance connection of the nebulae with the illuminating stars, the probability for any cloud to be illuminated by a star of some type, let us say A-type, will be equal to the total sum of volume illuminated by all A-type stars within the volume, divided by the whole volume $V$.

We are able to compute all these probabilities. If the hypothesis of the chance meeting is true, then the numbers of nebulae illuminated by stars of different types should be proportional to the corresponding probabilities.

The comparison of computed probabilities with the observed numbers of diffuse nebulae illuminated by stars of different types showed very close proportionality. We may conclude, therefore, that the hypothesis of chance meeting must be accepted.

These considerations lead also to another important consequence. It is easy to show that the stars of all types together illuminate only about $r / 2000$ of the volume of interstellar space. This means that a nebula has a probability of about $\mathrm{I} / 2000$ to be illuminated. 
It follows immediately that the number of all clouds in the part of the Galaxy accessible to our observations is about 2000 times larger than the number of the bright diffuse nebulae.

Following this line of argument we have established that the number of the cosmic clouds per $p s^{3}$ is about $\mathrm{I} / \mathrm{I0}, 000, \quad n \cong \mathrm{I} / \mathrm{I0}, 000$.

If $\sigma$ is the mean cross-section of a nebula, the number of clouds crossed by a ray along the path $l$ will be equal to $\ln \sigma$.

If, further, $\epsilon_{0}$ is the mean optical thickness of a cloud expressed in stellar magnitudes, the total absorption caused by these clouds will be

$$
\Delta m=l n \sigma \epsilon_{0}=a l,
$$

where $a$ is the mean absorption per parsec.

We know from the general data on the cosmic absorption the value of $a$ (photographic or visual) and the order of magnitude of the cross-section $\sigma$.

Therefore, if we assume that the whole interstellar absorption is caused by our system of clouds (or obscure nebulae), we may derive the value of $\epsilon_{0}$.

This first and very rough determination of $\epsilon_{0}$ showed that it is of the order of $0^{\mathbf{m} \cdot 2}$ or $\mathrm{o}^{\mathrm{m}} \cdot 3$ in the photographic region. It was clear that this value is not in contradiction to our ideas about the mean transparency of the diffuse nebulae.

It was concluded that the absorbing layer consists of a large number of discrete clouds, which are small compared with the distances between them. But it was desirable to have another, independent and more accurate method for the determination of $\epsilon_{0}$.

The counts of extragalactic nebulae made by Prof. Shapley and Dr Hubble have demonstrated that the numbers of nebulae brighter than a certain magnitude per square degree show considerable fluctuations. We have shown that even for a given galactic latitude these fluctuations far exceed the chance fluctuations according to Poisson's Law. It seems at first sight that this may be attributed to the clustering tendency, which of course exists and the importance of which was emphasized by Shapley.

However, the clustering tendency alone is not able to account for the main part of the fluctuations. This is particularly clear from the following evidence: when we divide the whole sky into galactic latitude zones and determine the fluctuations in each of these zones separately, the relative magnitude of these fluctuations increases with the decrease of the galactic latitude of the zone.

It is evident, however, that the chance fluctuations in the numbers of the galactic absorbing clouds on the path of light coming from the different extragalactic nebulae will cause additional fluctuations in nebulae numbers.

It remained to investigate theoretically how these fluctuations depend on the galactic latitude $b$.

With this aim in view, let us compute

$$
\overline{\left(N_{m}-\bar{N}_{m}\right)^{2}}={\overline{N_{m}^{2}}}_{m} \bar{N}_{m}^{2}
$$

where $N_{m}$ is the number of nebulae brighter than some $m$ per square degree. This number $N_{m}$ in the absence of absorption must be

$$
N_{m}=N_{0} .10^{0.6 m} \text {. }
$$

The transparency of a cloud is $q=I^{-\mathbf{0} \cdot 4 \epsilon_{0}}$. Therefore $n$ clouds in the line of sight diminish the brightness of nebulae $q^{n}$ times. The observable number of nebulae should therefore be

$$
N_{m}=N_{0} \cdot \mathrm{IO}^{0.6\left(m-r \epsilon_{0}\right)}=N_{0} \mathrm{IO}^{0.6 m} q^{\frac{3}{2} n} \text {. }
$$

The problem of the computation of $\overline{N_{m}}$ was reduced thus to the computation of $q^{\overline{i n}}$. At the same time the computation of $\overline{N_{m}^{2}}$ was reduced to the computation of $\overline{q^{3}}$. Using Poisson's Law for the probability of $n$ we have, after some algebra:

$$
\overline{N_{m}}=N_{0} . \mathrm{IO}^{0.6 \mathrm{6} m} e^{-n_{b}\left(1-q^{\bar{l}}\right)} \text {. }
$$


Here $n_{b}$ is the mean number of absorbing clouds crossed by the line of sight at the latitude $b$.

In the case of the plane parallel layers of clouds we have

$$
n_{b}=n_{\pi / 2} \operatorname{cosec} b \text {. }
$$

In the same way

$$
\overline{N_{m}^{2}}=N_{0}^{2} \mathrm{IO}^{1 \cdot 2 m} e^{-n_{b}\left(1-q^{3}\right)}
$$

and

$$
\frac{\overline{\left(N_{m}-\bar{N}_{m}\right)^{2}}}{\bar{N}_{m}^{2}}=e^{n_{b}\left(1-q^{\left.\frac{2}{2}\right)^{2}}\right.}-\mathrm{I}=e^{n} \pi / 2 \operatorname{cosec} b\left(1-q^{\frac{1}{2}}\right)^{2}-\mathrm{I}
$$

On the other hand, we have

$$
\tau_{\pi / 2}=n_{\pi / 2} \epsilon_{0}
$$

where $\tau_{\pi / 2}$ is the optical half thickness of the galactic absorbing layer in the direction perpendicular to the galactic plane. According to the last determination of Parenago $\tau_{\pi / 2}=0^{\mathrm{m}} \cdot 32$.

The equations (I) and (2) determine $n_{\pi / 2}$ and $\epsilon_{0}$. Using the counts of Shapley and Hubble we have computed the values of

$$
\frac{\left(N_{m}-\bar{N}_{m}\right)^{2}}{\bar{N}_{m}^{2}}
$$

for different latitudes and obtained a value of $\epsilon_{0}$ of the order of $0^{\mathrm{m}} \cdot 25$.

It should not be forgotten that it is necessary to introduce a correction accounting for the dispersion of the limiting magnitude of different plates and for other observational conditions. This correction is somewhat indefinite. However, it is still certain that $\epsilon_{0}$ is confined between

$$
\mathrm{O}^{\mathrm{m}} \cdot 2 \mathrm{O}<\epsilon_{0}<\mathrm{O}^{\mathrm{m}} \cdot 30 \text {. }
$$

It is clear from (2) that $n_{\pi / 2}$ will be of the order of unity.

Prof. Kukarkin has determined the dispersion of photoelectric colour-excesses of extragalactic nebulae in different latitudes and therefrom obtained immediately the mean colour-excess of one single cloud equal to $\mathrm{o}^{\mathrm{m}} \cdot 05$. Multiplying this value by the corresponding factor he has found for the optical thickness of a cloud the approximate value

$$
\epsilon_{0}=0^{\mathrm{m}} \cdot 27 \text {. }
$$

Dr Markaryan from Burakan Observatory has determined the value of $\epsilon_{0}$ from the comparison of the observed fluctuations in the numbers of the stars counted in low latitudes with the theory of fluctuations of star numbers in these latitudes based on the model of a layer of clouds as described above. His theory contains too much algebra to be exposed here. He obtained also $\epsilon_{0} \cong 0^{\mathrm{m}} \cdot 25$.

We may conclude that $\epsilon_{0}$ is really of this order, although we do not exclude that the value of $\epsilon_{0}$ may vary in different regions of our galaxy.

The theory of fluctuations in the total brightness of stars contained in a square degree in the galactic equator may take a very simple and elegant form. The distribution function of this quantity, which is nothing else than the intensity of the stellar component of the Milky Way radiation, satisfies a certain functional equation.

In the derivation of this equation I have used an invariance principle similar to that introduced by me in the theory of diffuse reflection from plane-parallel layers. More precisely, we have used the fact that the distribution function remains unchanged when the observer displaces himself by a distance $\Delta r$ along the line of sight.

This principle gives the following functional equation:

$$
\phi^{\prime}(J)+\phi(J)=\frac{\mathrm{I}}{q} \phi\left(\frac{J}{q}\right)
$$

for the distribution function, when $J$ is measured in some convenient units.

From the equation (3) it is easy to obtain for the mean-square deviation of intensity

$$
\frac{\overline{(J-\bar{J})^{2}}}{\bar{J}^{2}}=\frac{I-q}{I+q} \text {. }
$$


Unfortunately we have not at our disposal a sufficient number of determinations of $J$ at different points of the Milky Way to check the solution of (3) and the formula (4).

Some years ago, Academician Shajn called attention to the too weak correlation of the colour excesses of the B and O stars as determined by Stebbins and his co-workers, and the brightnesses of the corresponding regions of the Milky Way.

The theory of the cloud-structure of the absorbing layer explains at once this phenomenon. The stars of Stebbins's list have a mean distance above Iooo parsecs, while the fluctuations of the Milky Way brightness are caused chiefly by clouds at distances of 200-500 parsecs.

Therefore the sets of clouds responsible for these two phenomena are quite different and the correlation must be weak.

The problem under consideration is connected with the gas clouds also. Under the assumption that the interstellar gas has also such a patchy structure Dr Melnikov has analysed the curve of growth for the interstellar lines and obtained dispersion velocities of the gaseous clouds of the order of $8 \mathrm{~km} . / \mathrm{sec}$.

Dr Lyman Spitzer has told me that the observational data of W. S. Adams on the splitting of the interstellar lines into several components lead to a number of the gas clouds crossed by the line of sight equal to the number of the dust clouds computed from our theory. He identifies therefore the two systems of clouds. It follows from this identification that the clouds of interstellar gas should be comparatively small in size (about 8-Io parsecs in diameter). It is very important to prove this conclusion observationally.

In connection with the problem considered in this brief report a large amount of observational work is being done at the Abastumani Observatory under Dr Kharadze. The colour-indices of many thousands of stars in the selected areas are determined as well as the colours of many extragalactic nebulae.

However, the photo-electric data on the colour-indices of the external galaxies should be very important for the further study of the structure of the galactic absorbing layer.

\section{Discussion}

L. SPITZER, Jr. remarked that the very important work described by Ambartsumian cleared up two basic problems in the study of interstellar matter. In the first place the ratio of selective to general absorption found from the study of the external galaxies is the same as has been found by many workers for obscuring matter in the galactic plane. This removes the discrepancy found by Stebbins some time ago and emphasized by Seares. In the second place the close agreement between the number of obscuring clouds per kiloparsec found by Ambartsumian, by three quite independent methods, and the corresponding number of gas clouds per kiloparsec indicated by Adams's observations gives added confirmation to the picture of small, separate, randomly distributed clouds of gas and solid particles. This picture is in marked contrast to the previous one of a uniform interstellar medium. Spitzer concluded that to a first approximation the average characteristics of the interstellar clouds-size, separation, and general nature - now begin to be well established.

\section{ADAPTATION OF TWO-DIMENSIONAL CLASSIFICATION TO OBJECTIVE PRISM SPECTRA}

\section{By Miss M. WALTheR \\ Harvard College Observatory, Cambridge, Massachusetts}

A project has been undertaken at the Harvard Observatory to investigate the possibility of adapting a two-dimensional system of spectral classification, namely the Morgan-Keenan-Kellman system, to objective prism plates. Spectra obtained with an objective prism attached to the I2-inch Metcalf doublet are comparable in dispersion to 
those used by Morgan and his collaborators. However, it was necessary to focus special attention on the improvement of the quality of the objective prism spectra. Experimentation revealed that the Eastman Kodak emulsion IIa-O is superior to other available emulsions suitable for the project in question. Equally important is the development process used for the plates. Development in the Eastman Kodak developer DK 20 at $65^{\circ} \mathrm{F}$. for $\mathrm{I} 2$ to $\mathrm{I} 4$ minutes yields very satisfactory results.

For the purpose of establishing criteria for a two-dimensional classification, more than a hundred standard stars chosen from the Morgan-Keenan-Kellman Atlas were photographed with an objective-prism-telescope combination. From this material criteria for determining both spectral type and luminosity class have been established.

The fifteen regions chosen for classification include a number of Selected Areas of low galactic latitudes and fields which coincide with those studied at the Case Institute of Technology. The selection of these regions was made with the intent to use them as standard regions for which the Morgan-Keenan classification could be established.

A comparison of my classification with the Henry Draper classification is made for various magnitude intervals. For the stars brighter than $6 \mathrm{~m} \cdot 50$ there is essentially no systematic difference. For fainter stars systematic differences become more evident. At A o the two systems are in good agreement, but the following subtypes of the A stars are classified systematically earlier by Miss Cannon. The Henry Draper classification of the B stars, on the other hand, is later than the new Harvard classification, and the difference becomes more pronounced for successively fainter stars. The systematic differences for the later spectral types are relatively small.

The luminosity criteria established suffice to determine at least three luminosity classes for all spectral types except the late B stars and the A stars. A catalogue with luminosity classifications of approximately 600 stars has already been prepared for publication.

The dispersion and quality of the objective prism spectra are sufficiently high so that one may readily distinguish between the normal A stars and those which are called anomalous. These latter, belonging to two groups, the 'peculiar' and 'metallic-line' A stars, are found to occur quite frequently. The material examined thus far reveals 48 newly discovered anomalous A stars. From the data available it follows that $13 \%$ of the A stars in the magnitude interval, $5^{\mathrm{m} \cdot} \cdot 5^{\mathrm{O}}-9^{\mathrm{m}} \cdot 5^{\mathrm{O}}$, and at low galactic latitudes belong to the group of 'peculiar' A stars.

\section{Discussion}

Dr Gratton. The importance of a very accurate spectral classification, based on physically significant criteria is enormous. The Henry Draper catalogue is invaluable for studies on the distributions of the stars, but for studies on stellar structure it is almost useless. For instance, it cannot be used for tracing the fine structure features of the Hertzsprung-Russell diagram. Some years ago some doubts have been raised whether there exists or not a gap between giants and dwarfs for K-type stars. Using Mt Wilson or Kuiper unpublished observations of spectral types, one finds from the stars with trigonometric parallaxes larger than 0 " 050 a very well-defined main sequence with extremely small dispersion, besides nicely separated groups of giants and subdwarfs. With Harvard spectral types the dispersion around the main sequence is so large that these features are all obliterated; the gap between $\mathrm{Ko}_{\mathrm{O}}-\mathrm{K}_{2}$ giants and the main sequence is completely filled. 